\title{
Position-momentum entropic uncertainty relation and complementarity in single-slit and double-slit experiments
}

\author{
Jorge Sánchez-Ruiz \\ Departament de Física Fonamental, Universitat de Barcelona, Diagonal 647, 08028 Barcelona, Spain
}

\begin{abstract}
The exact analytical values of the position and momentum information entropies are calculated for the single slit and double slit diffraction experiments. In both cases, the product of the exponentials of the entro pies is strictly greater than the lower bound $\pi e \hbar$ given by the optimal entropic uncertainty relation for position and momentum, which implies that the single slit and double slit configurations are not minimum uncertainty states. The results obtained show that the position momentum entropic uncertainty relation provides a rigorous quantitative expression for the uncertainty principle in these experiments, unlike the Heisenberg inequality for standard deviations. However, it is also shown that, in the double slit experiment, wave particle duality cannot be derived from the entropic uncertainty relation.
\end{abstract}

PACS number(s): $03.65 . \mathrm{Bz}$

\section{INTRODUCTION}

In quantum mechanics, position and momentum components along one direction are a pair of complementary observables, satisfying the commutation rule $[X, P]=i \hbar$. As a consequence, the corresponding probability densities cannot both be arbitrarily concentrated, and, if one of them tends to a Dirac delta "function," the other one becomes more and more uniform. This impossibility of a precise simultaneous specification of position and momentum for a quantum object is the statement of the Heisenberg uncertainty principle $[1,2]$. Considering various gedanken experiments to determine the position and momentum of an electron, Heisenberg found that

$$
x_{1} p_{1} \approx \hbar
$$

where $x_{1}$ and $p_{1}$ are the "imprecisions" with which the values of $x$ and $p$ are determined [1]. A well-known example is the diffraction of a beam of light or particles by an infinitely long slit: for the diffracted particles, after passing through the slit, $x_{1}$ is given by the width of the slit, while $p_{1}$, as revealed by the diffraction pattern on a distant screen or photographic plate, is inversely proportional to the same width, so that the product of both uncertainties turns out to be a constant of the order of $\hbar$ (see Sec. II below).

In Ref. [1], Heisenberg did not give an exact definition for the "imprecisions" $x_{1}$ and $p_{1}$, but estimated them by some plausible measure in each case separately. It was Kennard [3] who proved for the first time the well-known inequality

$$
\Delta X \Delta P \geqslant \frac{\hbar}{2}
$$

where $\Delta A$ denotes the standard deviation of observable $A$, $(\Delta A)^{2}=\left\langle(A-\langle A\rangle)^{2}\right\rangle$. Equation (2) was soon adopted by Heisenberg as the true mathematical expression of the uncertainty principle for the position-momentum pair [2]. However, it has been pointed out [ 46$]$ that, in fact, this inequality fails to express adequately the physical contents of the uncertainty principle, as summarized by Eq. (1), in the case of the single-slit diffraction experiment. The same happens for Young's double-slit experiment, which has been used since the early times of quantum mechanics to illustrate wave-particle duality, i.e., the impossibility of observing both wavelike behavior and particlelike behavior of a quantum system in a single experiment [ $\left.\begin{array}{ll}7 & 9\end{array}\right]$.

An alternative mathematical formulation of the uncertainty principle is provided by the inequality $[10,11]$

$$
\delta X \delta P \geqslant \pi e \hbar,
$$

where $\delta A$ is defined as the exponential of the differential entropy corresponding to the observable $A$, with normalized probability density function $P(a)$,

$$
\delta A \equiv \exp \left(-\int P(a) \ln P(a) d a\right) .
$$

In the framework of Shannon's information theory [12], entropy is proved to be the only rigorous mathematical measure of the lack of knowledge or uncertainty associated to a random variable, so that entropic uncertainty relations such as Eq. (3) do properly express the physical contents of the uncertainty principle, that is, the impossibility of simultaneously having complete information about the values of a pair of observables with no common eigenstates. The quantity $\delta A$ defined in Eq. (4) has all the properties that we intuitively expect for a good measure of the width of a continuous probability distribution. In particular, $\delta A$ vanishes if and only if the probability density is a Dirac delta "function," and $\delta A=L$ for the uniform distribution over an interval of length $L$ in $A$ space, while a simple variational calculation shows that $\delta A<L$ for any nonuniform distribution vanishing outside that interval.

For an arbitrary one-dimensional probability density, we have the variational inequality [12]

$$
\Delta A \geqslant(2 \pi e)^{-1 / 2} \delta A \text {. }
$$

When combined with this result, the entropic uncertainty relation (3) yields the Heisenberg inequality (2), which proves the latter to be a consequence of the former. The sign of 
equality in Eq. (5) holds for Gaussian distributions, and the lower bound in Eq. (3) is attained for the ground state of the harmonic oscillator, where the probability densities for both position and momentum are Gaussian $[10,11]$, so that this is also the case for the lower bound in Eq. (2).

Equation (5) shows that standard deviation provides an upper bound for the entropic uncertainty measure defined by Eq. (4), and, for Gaussian distributions, as well as in many other cases, both quantities have the same order of magnitude. However, standard deviation can greatly overestimate the actual uncertainty, and it can even be infinite for distributions that decrease slowly enough for $x \rightarrow \infty$ but have a spread or extent as small as desired [5,6]. As we shall see below, this is what happens for the momentum probability densities of the diffracted particles in the one-slit and doubleslit experiments. The Heisenberg inequality (2) is then trivially satisfied for any finite value of $\Delta X$, and, by itself, does not forbid the simultaneous existence of arbitrarily narrow probability distributions of both position and momentum, which would violate the uncertainty principle [5].

The calculation of the entropic uncertainties $\delta X$ and $\delta P$ for physically interesting quantum states has been the subject of considerable effort in recent years (see, e.g., Refs. [13 16]). In the present paper, we obtain the exact analytical expression of these quantities for a quantum particle diffracted by a single slit (Sec. II) and a double slit (Sec. III). We thus show that, in both cases, the entropic uncertainty relation (3) is strong enough to provide a rigorous quantitative expression of the position-momentum uncertainty principle, unlike the Heisenberg inequality (2). Finally, in Sec. IV, we show that wave-particle duality cannot be derived from the entropic uncertainty relation in the double-slit experiment.

\section{ENTROPIC UNCERTAINTY RELATION IN THE SINGLE SLIT EXPERIMENT}

In the single-slit diffraction experiment, a monochromatic plane wave, representing an incoming beam of particles with momentum $p_{0}$, incides on a wall that contains an infinitely long slit of width $2 a$, and the diffracted particles are observed on a screen placed at a distance $l$ behind the slit. Without loss of generality, the wave functions in position and momentum spaces can be considered to be the Fourier pair:

$$
\psi(x)=\left\{\begin{array}{ll}
(2 a)^{-1 / 2} & \text { if }|x|<a, \\
0 & \text { if }|x|>a,
\end{array} \quad \phi(p)=\left(\frac{\hbar}{a \pi}\right)^{1 / 2} \frac{1}{p} \sin \left(\frac{a p}{\hbar}\right),\right.
$$

where $x$ is the position coordinate in the direction perpendicular to the slit, and $p$ is the momentum component along the same direction [5]. The most natural measure of the uncertainty in position is the width of the slit, $2 a$, while the intuitive value of the momentum uncertainty is $2 \pi \hbar / a$, the distance between the two central zeros of the momentum probability density $|\phi(p)|^{2}$ (see Fig. 1). This estimation for the uncertainty in momentum can also be obtained from the diffraction pattern that appears on the screen, since it can be shown that the wave function in position space at the screen, $\psi_{s}(x)$, is approximately equal to $\phi\left(p_{0} x / l\right)$, provided that the

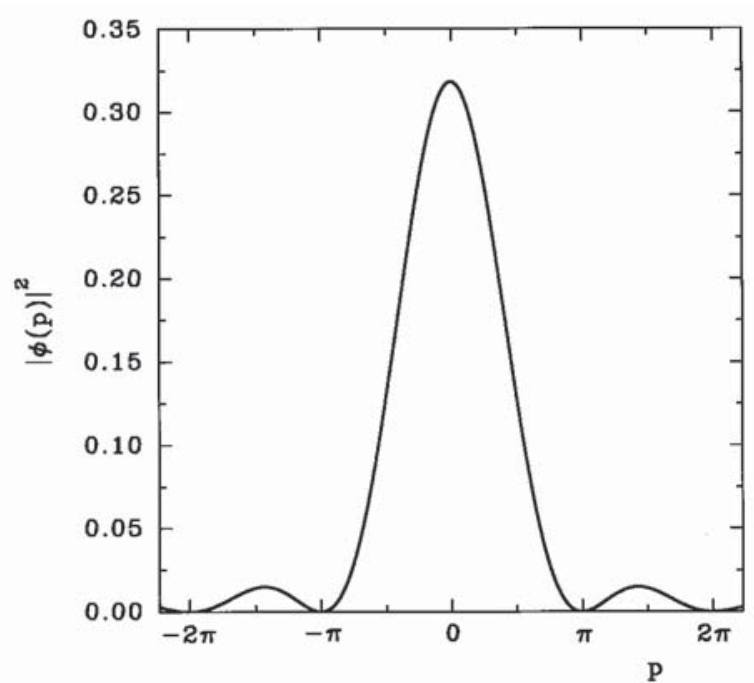

FIG. 1. Probability density of momentum for the single slit ex periment $(a / \hbar \equiv 1)$.

conditions for the so-called Fraunhofer diffraction $(l \gg a$ $\gg \hbar / p_{0}$ and $\left.l \gg|x|\right)$ are satisfied [5].

For the wave functions in Eq. (6), we have $\Delta X=a / \sqrt{3}$, which has the right order of magnitude (although it underestimates the actual uncertainty by a factor of $2 \sqrt{3} \approx 3.464$ ), but $\Delta P=\infty$, whatever the value of $a$ is. The divergence of $\Delta P$ is related to the discontinuity of $\psi(x)$ at the edges of the slit, and can be avoided by smoothing the sharp edges modeled by the spatial wave function of Eq. (6) over a small distance $b$, with $b \ll a$. However, $\Delta P$ then has a strong dependence on the value of $b$, and can be made arbitrarily large by taking $b$ small enough [5]. Thus we are led to the conclusion that, in this case, standard deviation is not an adequate measure for the uncertainty in momentum, and the inverse proportionality of position and momentum uncertainties, which is the basis of the uncertainty principle, cannot be deduced from Eq. (2).

On the other hand, the entropic uncertainties $\delta X$ and $\delta P$ are given by Eq. (4), with $P(x)=|\psi(x)|^{2}$ and $P(p)=|\phi(p)|^{2}$, respectively,

$$
\delta X=2 a,
$$

$$
\delta P=\frac{\pi \hbar}{a} \exp \left(-\frac{2}{\pi} I\right), \quad I \equiv \int_{0}^{\infty} \frac{\sin ^{2} x}{x^{2}} \ln \frac{\sin ^{2} x}{x^{2}} d x,
$$

so that they are inversely proportional to each other. Moreover, $\delta X$ coincides (exactly) with our previous estimation.

To complete the calculation of $\delta P$, we first write the integral $I$ in the form

$$
I=I_{A}-2 I_{B},
$$

$$
I_{A} \equiv \int_{0}^{\infty} \frac{\sin ^{2} x}{x^{2}} \ln \sin ^{2} x d x, \quad I_{B} \equiv \int_{0}^{\infty} \frac{\sin ^{2} x}{x^{2}} \ln x d x .
$$

The value of $I_{B}$ may be found in Refs. $[17,18]$, 


$$
I_{B}=-\frac{\pi}{2}(\gamma+\ln 2-1)
$$

where $\gamma$ is Euler's constant. As regards $I_{A}$, this integral can be simplified by taking advantage of the following result [18],

$$
\int_{0}^{\infty} \frac{f(\sin x)}{x^{2}} d x=\int_{0}^{\pi / 2} \frac{f(\sin x)}{\sin ^{2} x} d x, \quad f(-y)=f(y),
$$

which holds for any even function $f(y)$ such that the integrals in both sides do exist, and may be proved by substituting in the right-hand side the Mittag-Leffler development in elementary fractions [17]

$$
\frac{1}{\sin ^{2} x}=\sum_{n=-\infty}^{\infty} \frac{1}{(x+n \pi)^{2}} .
$$

Using Eq. (10), we get

$$
I_{A}=\int_{0}^{\pi / 2} \ln \sin ^{2} x d x=-\pi \ln 2
$$

which together with Eq. (9) leads to

$$
I=-\pi(1-\gamma)
$$
reads

Substituting for $I$ from Eq. (13) into (7), this equation

$$
\delta X=2 a, \quad \delta P=\frac{\pi \hbar}{a} \exp (2(1-\gamma))
$$

We see that $\delta P$ is very close to the intuitive value $2 \pi \hbar / a$, since $\exp (2(1-\gamma)) \approx 2.329$. The product of position and momentum entropic uncertainties,

$$
\delta X \delta P=2 \pi \hbar \exp (2(1-\gamma)),
$$

has the approximate value $1.714 \pi e \hbar$, thus satisfying inequality (3).

\section{ENTROPIC UNCERTAINTY RELATION IN THE DOUBLE SLIT EXPERIMENT}

In Young's double-slit experiment, a monochromatic plane wave incides on a wall containing two slits of width $2 a$, separated by a distance $2 d$ (we assume $d \geqslant a$, so that the slits do not overlap), and the resulting interference pattern is observed on a distant screen or photographic plate. As a simple model of the wave functions in position and momentum spaces for a double slit with sharp edges, we can take the Fourier pair [5]

$$
\psi(x)= \begin{cases}(4 a)^{-1 / 2} & \text { if } d-a<|x|<d+a, \\ 0 & \text { elsewhere }\end{cases}
$$

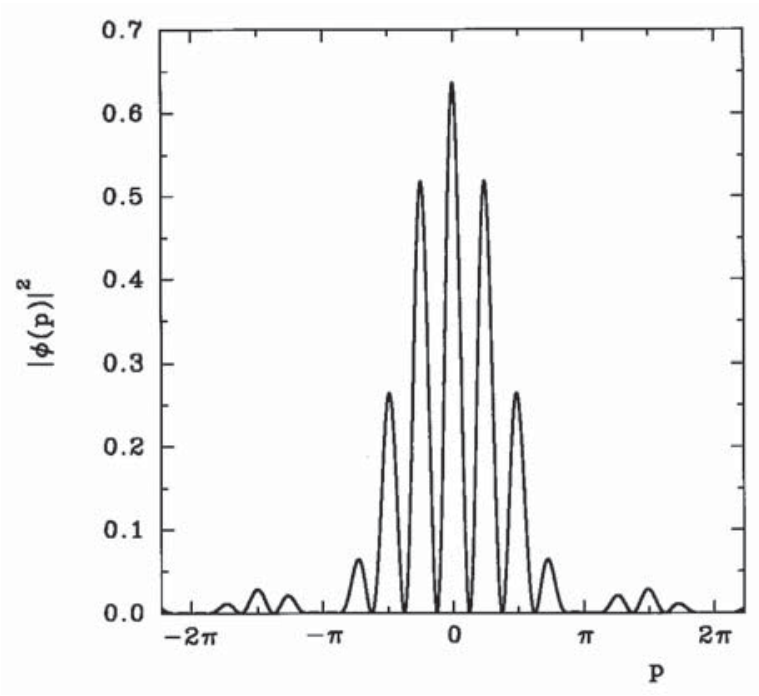

FIG. 2. Probability density of momentum for the double slit experiment $(a / \hbar \equiv 1, k=4)$.

$$
\phi(p)=\left(\frac{2 \hbar}{a \pi}\right)^{1 / 2} \frac{1}{p} \cos \left(\frac{d p}{\hbar}\right) \sin \left(\frac{a p}{\hbar}\right) .
$$

The momentum wave function $\phi(p)$ is related to the wave function in position space at the screen, $\psi_{s}(x)$, in the same way as in the single-slit case.

Reasonable estimations for the uncertainties in position and momentum are, respectively, $4 a$ and $2 \pi \hbar / a$ (from Fig. 2 we see that the envelope of $|\phi(p)|^{2}$ is the momentum probability density for the diffraction by a single slit displayed in Fig. 1). However, for the wave functions in Eq. (16), we have $\Delta X=\left(d^{2}+a^{2} / 3\right)^{1 / 2}$, which greatly overestimates the actual uncertainty for $d \gg a$, while $\Delta P=\infty$, whatever the values of $d$ and $a$ are. We thus see that standard deviation is not a good measure for the uncertainties in position and momentum, and, in the same way as in the singleslit case, Eq. (2) fails to capture the physical contents of the uncertainty principle.

The entropic uncertainties in position and momentum corresponding to the wave functions in Eq. (16) are

$$
\begin{gathered}
\delta X=4 a, \\
\delta P=\frac{\pi \hbar}{2 a} \exp \left(-\frac{4}{\pi} I(k)\right), \\
I(k) \equiv \int_{0}^{\infty} \frac{\cos ^{2} k x \sin ^{2} x}{x^{2}} \ln \frac{\cos ^{2} k x \sin ^{2} x}{x^{2}} d x,
\end{gathered}
$$

where we have introduced the convenient notation

$$
k \equiv \frac{d}{a}, \quad k \geqslant 1
$$

We see that the value of $\delta X$ is again equal to our previous intuitive estimation. 
Now we are faced with the problem of calculating $I(k)$ for $k \geqslant 1$. To achieve this goal, we first split $I(k)$ in the same way as $I(0)=I$ in the previous section [Eq. (8)],

$$
\begin{gathered}
I(k)=I_{1}(k)+I_{2}(k)-2 I_{3}(k), \\
I_{1}(k) \equiv \int_{0}^{\infty} \frac{\cos ^{2} k x \sin ^{2} x}{x^{2}} \ln \cos ^{2} k x d x, \\
I_{2}(k) \equiv \int_{0}^{\infty} \frac{\cos ^{2} k x \sin ^{2} x}{x^{2}} \ln \sin ^{2} x d x, \\
I_{3}(k) \equiv \int_{0}^{\infty} \frac{\cos ^{2} k x \sin ^{2} x}{x^{2}} \ln x d x .
\end{gathered}
$$

Making use of elementary trigonometric transformations, we can write

$$
\begin{aligned}
\cos ^{2} k x \sin ^{2} x= & \frac{1}{4}\left\{\sin ^{2}(k+1) x+\sin ^{2}(k-1) x+\cos 2 k x\right. \\
& -\cos 2 x\}
\end{aligned}
$$

Introducing this expression into the last formula of Eq. (19), and using the results $[17,18]$

$$
\int_{0}^{\infty} \frac{\sin ^{2} \mu x}{x^{2}} \ln x d x=-\frac{\pi \mu}{2}\{\gamma+\ln (2 \mu)-1\}, \quad \mu>0
$$

$$
\begin{aligned}
\int_{0}^{\infty} & \frac{\cos a x-\cos b x}{x^{2}} \ln x d x \\
\quad & =\frac{\pi}{2}\{(a-b)(\gamma-1)+a \ln a-b \ln b\}, \quad a>0, \quad b>0,
\end{aligned}
$$

the first of which is a generalization of Eq. (9), we get

$$
\begin{aligned}
I_{3}(k)= & \frac{\pi}{8}\{-2(\gamma-1+\ln 2)+2 k \ln k-(k+1) \ln (k+1) \\
& -(k-1) \ln (k-1)\}, \quad k \geqslant 1
\end{aligned}
$$

For integer values of $k, \cos ^{2} k x$ is an even function of $\sin x$, as may be seen from its finite product form [17]

$$
\begin{gathered}
\cos ^{2} k x=\frac{1}{2}+\frac{1}{2} \prod_{r=1}^{k}\left(1-\frac{\sin ^{2} x}{\sin ^{2}((2 r-1) \pi /(4 k))}\right), \\
k=1,2,3, \ldots,
\end{gathered}
$$

which enables us to calculate the integrals $I_{1}(k)$ and $I_{2}(k)$ by means of the same method that was used in the previous section for $I_{A}$. Equation (10), together with elementary properties of trigonometric functions and results in Refs. [17,18], leads to

$$
\begin{gathered}
I_{1}(k)=\int_{0}^{\pi / 2} \cos ^{2} k x \ln \cos ^{2} k x d x=\frac{1}{k} \int_{0}^{k \pi / 2} \cos ^{2} t \ln \cos ^{2} t d t=\int_{0}^{\pi / 2} \cos ^{2} t \ln \cos ^{2} t d t=-\frac{\pi}{4}(2 \ln 2-1), \\
I_{2}(k)=\int_{0}^{\pi / 2} \cos ^{2} k x \ln \sin ^{2} x d x=\int_{0}^{\pi / 2} \ln \sin x d x+\int_{0}^{\pi / 2} \cos 2 k x \ln \sin x d x=-\frac{\pi}{4}\left(2 \ln 2+\frac{1}{k}\right), \quad k=1,2,3, \ldots
\end{gathered}
$$

However, this procedure cannot be applied when $k$ is not an integer, so that we must use a different method in order to obtain a general expression for $I(k)$. Our new approach is based on the following result $[17,18]$ :

$$
\int_{0}^{\infty} \frac{\cos b x}{x^{2}+z^{2}} \ln \left(1-2 a \cos x+a^{2}\right) d x=\frac{\pi}{z}\left(\cosh (b z) \ln \left(1-a e^{-z}\right)+\sum_{n=1}^{[b]} \frac{a^{n}}{n} \sinh (z(b-n))\right)
$$

which is valid for $|a| \leqslant 1, b \geqslant 0, \operatorname{Re} z>0$ (here and in all the following formulas, the square brackets denote the integer part of the expression within). In the limiting cases $a= \pm 1$, taking into account that $2+2 \cos x=4 \cos ^{2}(x / 2), 2-2 \cos x=4 \sin ^{2}(x / 2)$, and $[17,18]$

$$
\int_{0}^{\infty} \frac{\cos b x}{x^{2}+z^{2}} d x=\frac{\pi}{2 z} e^{-b z}, \quad z>0, \quad b \geqslant 0
$$

Eq. (25) yields

$$
\begin{gathered}
\int_{0}^{\infty} \frac{\cos b x}{x^{2}+z^{2}} \ln \cos ^{2} \frac{x}{2} d x=\frac{\pi}{z}\left(\cosh (b z) \ln \left(1+e^{-z}\right)-e^{-b z} \ln 2+\sum_{n=1}^{[b]} \frac{(-1)^{n}}{n} \sinh (z(b-n))\right), \\
\int_{0}^{\infty} \frac{\cos b x}{x^{2}+z^{2}} \ln \sin ^{2} \frac{x}{2} d x=\frac{\pi}{z}\left(\cosh (b z) \ln \left(1-e^{-z}\right)-e^{-b z} \ln 2+\sum_{n=1}^{[b]} \frac{1}{n} \sinh (z(b-n))\right) .
\end{gathered}
$$

Making the appropriate changes of variables, these integrals can be cast into the equivalent form 


$$
\begin{gathered}
\int_{0}^{\infty} \frac{\cos b x}{x^{2}+z^{2}} \ln \cos ^{2} k x d x=\frac{\pi}{z}\left(\cosh (b z) \ln \left(1+e^{-2 k z}\right)-e^{-b z} \ln 2+\sum_{n=1}^{[b /(2 k)]} \frac{(-1)^{n}}{n} \sinh (z(b-2 n k))\right) \\
\int_{0}^{\infty} \frac{\cos b x}{x^{2}+z^{2}} \ln \sin ^{2} x d x=\frac{\pi}{z}\left(\cosh (b z) \ln \left(1-e^{-2 z}\right)-e^{-b z} \ln 2+\sum_{n=1}^{[b / 2]} \frac{1}{n} \sinh (z(b-2 n))\right)
\end{gathered}
$$

The next step is taking the limit $z \rightarrow 0$ in Eq. (28). Using the well-known Taylor series expansions for exponential, hyperbolic, and logarithmic functions [17], we find

$$
\begin{aligned}
& \int_{0}^{\infty} \frac{\cos b x}{x^{2}+z^{2}} \ln \cos ^{2} k x d x=\pi F_{k}(b)+O(z), \\
& \int_{0}^{\infty} \frac{\cos b x}{x^{2}+z^{2}} \ln \sin ^{2} x d x=\pi G_{z}(b)+O(z),
\end{aligned}
$$

where the functions $F_{k}(b)$ and $G_{z}(b)$ are defined as

$$
\begin{gathered}
F_{k}(b) \equiv-k+b \ln 2+\sum_{n=1}^{[b /(2 k)]} \frac{(-1)^{n}}{n}(b-2 n k), \\
G_{z}(b) \equiv \frac{\ln z}{z}-1+b \ln 2+\sum_{n=1}^{[b / 2]} \frac{1}{n}(b-2 n) .
\end{gathered}
$$

Writing the trigonometric identity (20) in the alternative form

$$
\cos ^{2} k x \sin ^{2} x=\frac{1}{4}\left(1+\cos 2 k x-\cos 2 x-\frac{1}{2} \cos 2(k+1) x-\frac{1}{2} \cos 2(k-1) x\right),
$$

the integrals $I_{1}(k)$ and $I_{2}(k)$ can be expressed in terms of $F_{k}(b)$ and $G_{z}(b)$ as follows:

$$
\begin{gathered}
I_{1}(k)=\frac{\pi}{4}\left(F_{k}(0)-F_{k}(2)+F_{k}(2 k)-\frac{1}{2} F_{k}(2(k+1))-\frac{1}{2} F_{k}(2(k-1))\right), \\
I_{2}(k)=\frac{\pi}{4} \lim _{z \rightarrow 0}\left(G_{z}(0)-G_{z}(2)+G_{z}(2 k)-\frac{1}{2} G_{z}(2(k+1))-\frac{1}{2} G_{z}(2(k-1))\right) .
\end{gathered}
$$

After some algebra, Eq. (32) simplifies to

$$
I_{1}(k)=-\frac{\pi}{4}(2 \ln 2-1), \quad I_{2}(k)=-\frac{\pi}{4}\left(2 \ln 2+\frac{1}{[k]}-\frac{k-[k]}{[k]([k]+1)}\right), \quad k \geqslant 1,
$$

which is the generalization of Eq. (24) to arbitrary values of $k$ (the condition on $k$ arises from $b \geqslant 0$ ). In particular, we have found the somewhat surprising result that the value of $I_{1}(k)$ is independent of $k$ for $k \geqslant 1$.

Equations (22) and (33) provide us with an explicit expression for the integral $I(k)$ in the first line of Eq. (19),

$$
I(k)=-\frac{\pi}{4}\left(1-2 \gamma+2 \ln 2+\frac{1}{[k]}-\frac{k-[k]}{[k]([k]+1)}+2 k \ln k-(k+1) \ln (k+1)-(k-1) \ln (k-1)\right),
$$

and substitution for $I(k)$ from Eq. (34) into Eq. (17) gives

$$
\delta X=4 a, \quad \delta P=\frac{2 \pi \hbar}{a} \frac{k^{2 k}}{(k+1)^{k+1}(k-1)^{k-1}} \exp \left(1-2 \gamma+\frac{1}{[k]}-\frac{k-[k]}{[k]([k]+1)}\right) .
$$

The product of position and momentum entropic uncertainties,

$$
\delta X \delta P=8 \pi \hbar \frac{k^{2 k}}{(k+1)^{k+1}(k-1)^{k-1}} \exp \left(1-2 \gamma+\frac{1}{[k]}-\frac{k-[k]}{[k]([k]+1)}\right),
$$




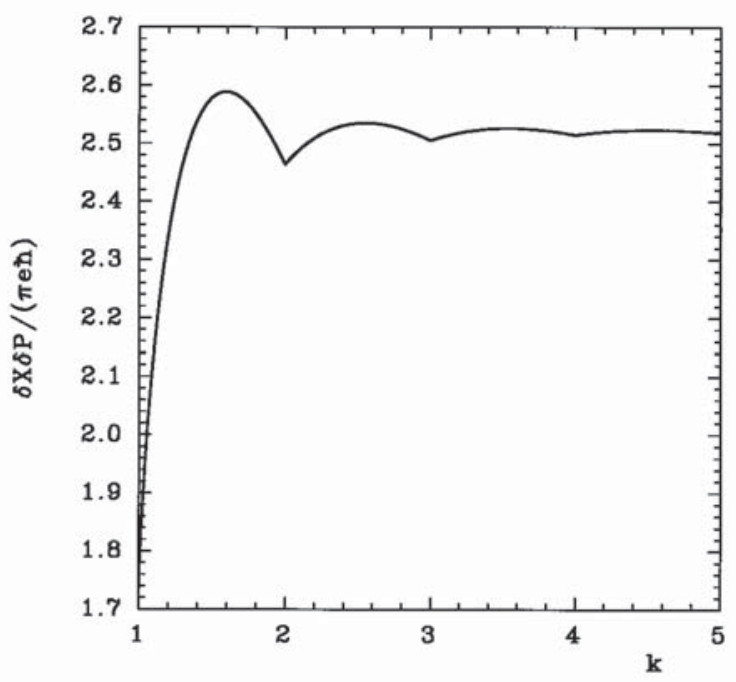

FIG. 3. Product of the entropic uncertainties in position and momentum for the double slit experiment $(1 \leqslant k \leqslant 5)$.

is displayed as a function of $k$ in Fig. 3. We see that it presents an oscillating behavior, with local minima at integer values of $k$, the first of which $(k=1)$ is the absolute minimum, and local maxima between consecutive minima. The absolute maximum is again the first one, and, if $k_{n}$ denotes the maximum satisfying $n<k_{n}<n+1 \quad(n=1,2,3, \ldots)$, a simple calculation using Eq. (34) shows that

$$
k_{n}=\left\{1-\exp \left(-\frac{1}{n(n+1)}\right)\right\}^{-1 / 2} \text {. }
$$

The product $\delta X \delta P$ is thus bounded below by its value at $k=1,2 \pi \hbar \exp (2(1-\gamma)) \approx 1.714 \pi e \hbar$. The fact that this value coincides with that obtained in the previous section for the single-slit experiment, Eq. (15), is not surprising since $k=1$ is equivalent to $d=a$, which means that in this case we actually have only one slit, of width $4 a$. As regards the upper bound, which is attained for $k=k_{1}$ $=\left(1-e^{-1 / 2}\right)^{-1 / 2} \approx 1.594$, its approximate numerical value is $2.590 \pi e \hbar$. The amplitude of the oscillations decreases as $k$ increases, and, in the limit $k \rightarrow \infty, \delta X \delta P$ tends to $8 \pi \hbar \exp (1-2 \gamma) \approx 2.522 \pi e \hbar$. We also see from Fig. 3 that $\delta X \delta P \approx 8 \pi \hbar \exp (1-2 \gamma)$ unless the value of $k$ is very low.

\section{DISCUSSION}

In summary, we have calculated the exact analytical values of the entropic uncertainties in position and momentum for the diffracted particles in the single-slit and double-slit experiments. Our results, Eqs. (14) and (35), show that the entropic uncertainty relation (3), unlike the Heisenberg inequality for standard deviations (2), provides a true quantitative expression for the position-momentum uncertainty principle in these experiments, which enables us to lay on a rigorous foundation the qualitative arguments that were used by Heisenberg in his original formulation of the uncertainty principle [1,2]. Equations (14) and (35) also show that the single-slit and double-slit configurations are not states of minimum uncertainty with respect to the entropic definition adopted in this paper.

In Young's double-slit experiment, wave-particle duality is displayed by the fact that the determination of which slit a photon (or electron) arriving to the screen has passed through is incompatible with the appearance of the interference pattern on the screen [9]. In many experimental situations, such as the famous recoiling-slit gedanken experiment discussed by Einstein and Bohr [8], wave-particle duality can be considered as a consequence of the position-momentum uncertainty principle, since any attempt to precisely determine the position of the particle at the wall containing the slits introduces an uncertainty in momentum that suffices to destroy the interference pattern [9]. However, recent advances in the field of quantum optics have led to the proposal of experimental procedures that allow "which-path" detection without disturbing the particles, and the issue of whether or not wave-particle duality is always enforced by the uncertainty principle has become the subject of an intense controversy [19].

In the classic discussions of the double-slit experiment [7 9], the uncertainty principle is always used in the qualitative, order-of-magnitude form (1), because of the inadequacy of the rigorous quantitative formulation provided by Eq. (2). On the other hand, Shannon's entropy has been used by Wootters and Zurek to give a quantitative formulation of wave-particle duality in the double-slit experiment [20]. Thus a natural question that arises at this stage is whether wave-particle duality can be derived from the entropic inequality (3). As we shall see in the following, the answer appears to be in the negative.

Let us assume that a measurement is performed on the diffracted particles, whose entropic uncertainties $\delta X$ and $\delta P$ are originally given by Eq. (35), such as a complete "whichpath" determination is achieved. If the position and momentum entropic uncertainties corresponding to the postmeasurement state of the particles are denoted by $\delta X^{\prime}$ and $\delta P^{\prime}$, respectively, we shall have $\delta X^{\prime} \leqslant 2 a=\delta X / 2$ [see the comments after Eq. (4)]. On the other hand, if the interference pattern is maintained, the momentum distribution will remain the same, and, in particular, $\delta P^{\prime}=\delta P$. Therefore, $\delta X^{\prime}$ and $\delta P^{\prime}$ should satisfy the condition $\delta X^{\prime} \delta P^{\prime} \leqslant \delta X \delta P / 2$, in addition to Eq. (3). Combination of these two conditions yields the inequality $\delta X \delta P \geqslant 2 \pi e \hbar$, which from Fig. 3 is readily shown to hold for $k \geqslant k_{0}$, where $k_{0} \approx 1.057$ is the only solution of the equation $\delta X \delta P=2 \pi e \hbar$. Taking into account that $1 \leqslant k_{0} \leqslant 2$, as may be seen from Fig. 3, the equation defining $k_{0}$ can be written as

$$
\frac{4 k_{0}^{2 k_{0}}}{\left(k_{0}+1\right)^{k_{0}+1}\left(k_{0}-1\right)^{k_{0}-1}} \exp \left(-2 \gamma+\frac{3-k_{0}}{2}\right)=1 \text {. }
$$

We thus conclude that, leaving aside the case when $k<k_{0}$ (it is usually assumed that $d \gg a$, which is equivalent to $k \gg 1$ ), the position-momentum entropic uncertainty relation (3) is too weak to forbid the existence of a quantum state displaying both "which-slit" determination and the premeasurement interference pattern. The cause of this failure may 
be traced back to the properties of the uncertainty measures $\delta X$ and $\delta P$. Bohr's argument concerning the relation between wave-particle duality and the uncertainty principle $[5,7,8]$ rests on the inverse proportionality of the distance between the slits, $d$, and the width of the interference bands (or, equivalently, the width of the peaks of the momentum distribution displayed in Fig. 2), which is roughly given by $\hbar / d$. However, as we have found in the previous section, for the double-slit configuration $\delta X$ depends only on the sum of the widths of the two slits and not on the distance between them, while $\delta P$ measures the overall spread of the interference pattern but is insensitive to its fine structure. In this respect, it is interesting to note that Uffink and Hilgevoord [5] have proposed a quantitative expression of the uncer- tainty principle for position and momentum that does imply wave-particle duality when it is applied to the double-slit experiment; however, their relation makes use of different kinds of uncertainty measures for each observable, a measure of overall width for position and a measure of fine structure width (mean peak width) for momentum, so that it is not a true uncertainty relation in the same sense as Eqs. (1), (2), or (3).

\section{ACKNOWLEDGMENT}

The author gratefully acknowledges the financial support of the Fundació Aula, Barcelona, Spain.
[1] W. Heisenberg, Z. Phys. 43, 172 (1927).

[2] W. Heisenberg, The Physical Principles of the Quantum Theory (University of Chicago Press, Chicago, 1930).

[3] E. H. Kennard, Z. Phys. 44, 326 (1927).

[4] G. Beck and H. M. Nussenzweig, Nuovo Cimento 9, 1068 (1958).

[5] J. B. M. Uffink and J. Hilgevoord, Found. Phys. 15, 925 (1985); J. Hilgevoord and J. B. M. Uffink, Eur. J. Phys. 6, 165 (1985).

[6] J. B. M. Uffink, Ph.D. thesis, University of Utrecht, 1990.

[7] N. Bohr, Naturwissenschaften 16, 245 (1928); Nature (Lon don) 121, 580 (1928).

[8] N. Bohr, in Albert Einstein: Philosopher Scientist, edited by P. A. Schilpp (Library of Living Philosophers, Evanston, 1949); reprinted in Quantum Theory and Measurement, edited by J. A. Wheeler and W. H. Zurek (Princeton University Press, Princeton, NJ, 1983).

[9] R. P. Feynman, R. B. Leighton, and M. Sands, The Feynman Lectures on Physics (Addison Wesley, Reading, MA, 1965), Vol. III.

[10] W. Beckner, Ann. Math. 102, 159 (1975).

[11] I. Bialynicki Birula and J. Mycielski, Commun. Math. Phys. 44, 129 (1975).
[12] C. E. Shannon, in The Mathematical Theory of Communica tion, edited by C. E. Shannon and W. Weaver (University of Illinois Press, Urbana, 1949).

[13] R. J. Yáñez, W. Van Assche, and J. S. Dehesa, Phys. Rev. A 50, 3065 (1994).

[14] W. Van Assche, R. J. Yáñez, and J. S. Dehesa, J. Math. Phys. 36, 4106 (1995).

[15] V. Majerník and T. Opatrný, J. Phys. A 29, 2187 (1996).

[16] J. Sánchez Ruiz, Phys. Lett. A 226, 7 (1997).

[17] I. S. Gradshteyn and I. M. Ryzhik, Table of Integrals, Series, and Products (Academic Press, Boston, 1994), 5th ed.

[18] A. P. Prudnikov, Yu. A. Brychkov, and O. I. Marichev, Inte grals and Series. Volume 1: Elementary Functions (Gordon and Breach, New York, 1986).

[19] M. O. Scully, B. G. Englert, and H. Walther, Nature (London) 351, 111 (1991); P. Storey, S. Tan, M. Collett, and D. F. Walls, ibid. 367, 626 (1994); B. G. Englert, M. O. Scully, and H. Walther, ibid. 375, 367 (1995); E. P. Storey, S. M. Tan, M. J. Collett, and D. F. Walls, ibid. 375, 368 (1995).

[20] W. K. Wootters and W. H. Zurek, Phys. Rev. D 19, 473 (1979). 